Dossier: Mujeres y Educación en la Universidad Nacional

Red de Mujeres Investigadoras de la Universidad Nacional (UNA)

\title{
Mujeres en condición de interinazgo, la lucha por la estabilidad laboral y el crecimiento profesional en la UNA, 2020
}

\author{
Maritza Rodríguez Soto \\ Universidad Nacional de Costa Rica, Costa Rica \\ maritza.rodriguez.soto@una.ac.cr \\ https://orcid.org/0000-0002-7083-5260 \\ Laura Solís Bastos \\ Universidad Nacional de Costa Rica, Costa Rica \\ laura.solis.bastos@una.ac.cr \\ https://orcid.org/0000-0002-7434-221X
}

Recibido: 4 de agosto de 2020

Aceptado: 10 de noviembre de 2020

Resumen: El objetivo del artículo es presentar los resultados del censo sobre interinazgo en la Universidad Nacional a partir de las respuestas brindadas por mujeres administrativas o académicas interinas de las Facultades de Ciencias Sociales y Filosofía y Letras. Los datos recopilados forman parte del proyecto de investigación Implicaciones de la condición de interinazgo en la subjetividad y relaciones vinculares de población trabajadora de la UNA, adscrito al Programa Umbral Político, del Instituto de Estudios Sociales en Población.

A partir de las respuestas brindadas por las mujeres en condición de interinazgo, se obtiene una serie de resultados vinculados a las condiciones laborales y el crecimiento profesional de estas funcionarias. Se evidencia que el ser una persona contratada en condición de interino/a significa una vulnerabilidad para el crecimiento profesional y personal, además el ser mujer, las coloca en una condición de doble inferioridad ante las limitaciones estructurales instauradas por la sociedad patriarcal.

\section{(C) $(0 \odot$}

La Revista Estudios es editada por la Universidad de Costa Rica y se distribuye bajo una Licencia Creative Commons Atribución-NoComercial-CompartirIgual 3.0 Costa Rica. Para más información envíe un mensaje a 
Dossier: Mujeres y Educación en la Universidad Nacional

Red de Mujeres Investigadoras de la Universidad Nacional (UNA)

Palabras clave: Interinazgo; desigualdad de género; crecimiento laboral; crecimiento profesional; educación universitaria

\section{Women in the fight for job stability and professional growth at UNA, 2020}

Abstract: The objective of the article is to present the results of the census about indefinitely hired population at the National University, based on the answers provided by interim administrative or academic women at Faculties of Social Sciences and Philosophy and Letters. The data collected is part of the research project "Implications of indefinitely hired in the subjectivity and relationship of the working population of UNA", attached to the Political Threshold Program of the Institute for Social Studies in Population.

Based on the answers provided by indefinitely hired women, a series of results are obtained related to working conditions and the professional growth. It is evident that being a person hired as an interim means a vulnerability for professional and personal growth, in addition being a woman places them in a condition of double vulnerability to the structural limitations established by the patriarchal society.

Keywords: indefinitely hired; gender inequality; job growth; professional growth; university education

\section{Introducción}

El artículo da a conocer resultados del censo dirigido a población interina como parte de las acciones del proyecto de investigación denominado Implicaciones de la condición de interinazgo en la subjetividad y relaciones vinculares de población trabajadora de la Universidad Nacional, que se lleva a cabo desde el Programa Umbral Político, del Instituto de Estudios Sociales en Población (IDESPO-UNA), en lo que corresponde a la situación que viven las mujeres en condición de interinazgo, la lucha por alcanzar la estabilidad laboral y el crecimiento profesional.

\section{(C) $(0 \odot$}

La Revista Estudios es editada por la Universidad de Costa Rica y se distribuye bajo una Licencia Creative Commons Atribución-NoComercial-CompartirIgual 3.0 Costa Rica. Para más información envíe un mensaje a 
Dossier: Mujeres y Educación en la Universidad Nacional

Red de Mujeres Investigadoras de la Universidad Nacional (UNA)

El censo corresponde a la II fase de la investigación cuyo propósito es conocer el sentir de la población contratada, tanto académica como administrativa, nombrada en forma interina en una jornada de al menos medio tiempo semanal, con un mínimo de cinco años de laborar en la institución, en las Facultades de Ciencias Sociales y Filosofía y Letras, respecto a las implicaciones en la subjetividad y relaciones vinculares de esta forma de contratación laboral.

Para la Universidad Nacional, el nombramiento interino también denominado recientemente como "no propietario", es una forma de contratación que mantiene la población funcionaria, tanto administrativa como académica, que se caracteriza por no tener una relación laboral en propiedad, obtenida mediante concurso, lo que tiene implicaciones para las personas trabajadoras y sus familias dado que no son nombramientos permanentes. Esta condición laboral, denominada interinazgo, ha sido poco estudiada, no obstante, en la Universidad Nacional existen estudios referentes a esta temática como la investigación de Gamboa (2015), acerca del síndrome de desgaste por el trabajo y la precarización laboral en funcionarios y funcionarias con nombramiento interino de la Universidad Nacional; Alfaro y Alvarado (2012) mediante su trabajo final de graduación estudiaron el proceso de atracción, selección y vinculación del personal académico interino. Otra referencia para este tema es el estudio de Fernández, Jiménez, Marenco y Sequeira (2014) investigadoras del Instituto de Estudios de la Mujer (IEM) de la Universidad Nacional, quienes realizaron un diagnóstico acerca de las relaciones de equidad entre mujeres y hombres de la UNA, y por último la investigación realizada por Segura (2014), la cual investigó, desde un enfoque cualitativo, sobre el acoso laboral y psicológico en la UNA, en un estudio de casos denunciados durante el 2008 y 2009.

\footnotetext{
(ब) $\odot \Theta($

La Revista Estudios es editada por la Universidad de Costa Rica y se distribuye bajo una Licencia Creative Commons Atribución-NoComercial-CompartirIgual 3.0 Costa Rica. Para más información envíe un mensaje a revistaestudios.eeg@ucr.ac.cr.
} 
Dossier: Mujeres y Educación en la Universidad Nacional

Red de Mujeres Investigadoras de la Universidad Nacional (UNA)

Estos estudios arrojan información de relevancia, por ejemplo, las investigadoras del IEM (Fernández et al, 2014), señalan que "Es evidente que la contratación de personas interinas constituye, a todas luces, un problema ético que contraviene principios de igualdad, solidaridad y humanismo, inaceptables en la Universidad que ha definido esos valores como orientadores de su quehacer" (p.150). Por su lado Gamboa (2015) identificó que algunas de las personas trabajadoras en condición de interinazgo tienden aceptar condiciones como el retraso salarial y sobrecarga laboral, los cuales se vinculan a formas de precarización del trabajo.

Los resultados de estas investigaciones denotan la importancia y necesidad de dar seguimiento, a través de otros estudios, a la contratación interina dado que refleja un problema estructural.

En concordancia con estos estudios anteriormente citados, se reitera la importancia de dar continuidad al análisis de las condiciones laborales de la población interina, razón por la cual surge el proyecto de investigación Implicaciones de la condición de interinazgo en la subjetividad y relaciones vinculares de población trabajadora de la Universidad Nacional. A través de este artículo se pretende dar a conocer el caso particular de las funcionarias administrativas y académicas, y las repercusiones que enfrentan como personal interino/no propietario para propiciar espacios de reflexión acerca de esta la realidad laboral e institucional, que permitan generar cambios para el bienestar integral de la población trabajadora interina de la universidad.

\section{(c) (i) (9) (2)}

La Revista Estudios es editada por la Universidad de Costa Rica y se distribuye bajo una Licencia Creative Commons Atribución-NoComercial-CompartirIgual 3.0 Costa Rica. Para más información envíe un mensaje a 
Dossier: Mujeres y Educación en la Universidad Nacional

Red de Mujeres Investigadoras de la Universidad Nacional (UNA)

\section{Metodología}

En lo que respecta a la metodología de investigación de la ll fase de la investigación, es de tipo cuantitativa, se realizó un censo, mediante un cuestionario, a la población de funcionarios y funcionarias en condición de interinazgo de la Facultad de Ciencias Sociales y la Facultad de Filosofía y Letras de la Universidad Nacional. Este registro está compuesto por un total de 101 personas, que tenían al menos cinco años de laborar para la institución, una jornada mínima de medio tiempo, y contar con la condición de interinazgo al momento de selección de la muestra. De esta población, 74 personas contestaron el cuestionario, de ellas 35 mujeres y 39 hombres. El cuestionario digital fue aplicado de junio a agosto del año 2019, se podía contestar vía telefónica o de forma presencial, y contemplaba diferentes ámbitos referentes a la condición laboral del interinazgo, entre estas, implicaciones en el ámbito laboral, familiar, de género y a nivel económico. El análisis de los resultados obtenidos se lleva a cabo desde una perspectiva de género, considerando elementos teóricos de gran relevancia como las brechas laborales, división sexual del trabajo y el techo de cristal.

\section{Resultados}

En este apartado se presentan los principales resultados referentes a las características de la población participante en el censo, así como la realidad que viven mujeres en condición de interinazgo, su lucha por alcanzar la estabilidad laboral y el crecimiento profesional en la Universidad Nacional.

\section{(c) (i) (2) (2)}

La Revista Estudios es editada por la Universidad de Costa Rica y se distribuye bajo una Licencia Creative Commons Atribución-NoComercial-CompartirIgual 3.0 Costa Rica. Para más información envíe un mensaje a 
Dossier: Mujeres y Educación en la Universidad Nacional

Red de Mujeres Investigadoras de la Universidad Nacional (UNA)

\section{Características de la población participante en el censo.}

La tabla 1 muestra que de las 74 personas interinas que contestaron el cuestionario $47.3 \%$ representan el sexo femenino y $52.7 \%$ el masculino.

Tabla 1. Sexo de la población interina censada.

\begin{tabular}{|l|r|}
\hline Sexo & \% de la población censada \\
\hline Mujer & $47.3 \%$ \\
\hline Hombre & $52.7 \%$ \\
\hline Total & $\mathbf{1 0 0 , 0}$ \\
\hline
\end{tabular}

Fuente: IDESPO-UNA. Censo Percepciones sobre las condiciones de interinazgo en la Universidad Nacional, 2020. Elaboración propia.

Respecto al estamento de la población, tal y como lo muestra la figura 1, un $40 \%$ de las mujeres son administrativas y un $60 \%$ son académicas. En el caso de los hombres, $5,1 \%$ son administrativos y $94,9 \%$ académicos. Lo anterior, indica que las mujeres participantes en el censo ocupan más puestos administrativos que los hombres.

\section{(C) $(0 \odot$}

La Revista Estudios es editada por la Universidad de Costa Rica y se distribuye bajo una Licencia Creative Commons Atribución-NoComercial-CompartirIgual 3.0 Costa Rica. Para más información envíe un mensaje a revistaestudios.eeg@ucr.ac.cr. 
Dossier: Mujeres y Educación en la Universidad Nacional Red de Mujeres Investigadoras de la Universidad Nacional (UNA)

Figura 1. Estamento de la población participante en el censo, según cargo y sexo.

\section{Funcionarias}

\section{Funcionarios}

\section{$40 \%$ administrativas} $60 \%$ académicas

\section{$5,1 \%$ administrativos 94,9\% académicos}

Fuente: IDESPO-UNA. Censo Percepciones sobre las condiciones de interinazgo en la Universidad Nacional, 2020. Elaboración propia.

En lo que concierne a las edades en años cumplidos, la tabla 2 muestra que una mayoría de las personas tienen edades entre los 30 y 39 años, no obstante, existen funcionarias/os con 60 o más años de edad, que se encuentran en condición de interinazgo, cercanas a su jubilación, expuestas a la vulnerabilidad que esta condición laboral implica, lo que podría afectar las cotizaciones a su régimen de pensiones.

Tabla 2. Rango de edades en años cumplidos de la población censada, 2020.

\begin{tabular}{|l|r|}
\hline EDAD & $\%$ \\
\hline $30-39$ & 37,1 \\
\hline $40-49$ & 29,0 \\
\hline $50-59$ & 28,6 \\
\hline 60 y más & 5,7 \\
\hline Total & $\mathbf{1 0 0 , 0}$ \\
\hline
\end{tabular}

Fuente: IDESPO-UNA. Censo Percepciones sobre las condiciones de interinazgo en la Universidad Nacional, 2020. Elaboración propia.

\section{(C) $(\Theta \odot$}

La Revista Estudios es editada por la Universidad de Costa Rica y se distribuye bajo una Licencia Creative Commons Atribución-NoComercial-CompartirIgual 3.0 Costa Rica. Para más información envíe un mensaje a revistaestudios.eeg@ucr.ac.cr. 
Dossier: Mujeres y Educación en la Universidad Nacional

Red de Mujeres Investigadoras de la Universidad Nacional (UNA)

En lo referente al estado civil de las funcionarias participantes en el censo, la tabla 3 refiere que $33,3 \%$ de académicas se encuentran casadas así como un $57.1 \%$ de administrativas. Existe una mayor tendencia, $23,8 \%$ de las académicas a vivir en unión libre o de hecho con sus parejas contrario a un 7,1\% de las administrativas. De igual manera, un $28,6 \%$ de las académicas se encuentran divorciadas respecto a un $14,3 \%$ de administrativas.

Tabla 3. Estado civil de las funcionarias censadas, 2020.

\begin{tabular}{|l|r|r|}
\hline & Funcionarias Académicas & $\begin{array}{r}\text { Funcionarias } \\
\text { Administrativas }\end{array}$ \\
\hline Unión libre & $23,8 \%$ & $7,1 \%$ \\
\hline Casada & $33,3 \%$ & $57,1 \%$ \\
\hline Divorciada & $28,6 \%$ & $14,3 \%$ \\
\hline Viuda & $4,8 \%$ & $21,4 \%$ \\
\hline Soltera & $9,5 \%$ & $\mathbf{1 0 0 , 0} \%$ \\
\hline Total & $\mathbf{1 0 0 , 0 \%}$ & \\
\hline
\end{tabular}

Fuente: IDESPO-UNA. Censo Percepciones sobre las condiciones de interinazgo en la Universidad Nacional, 2020. Elaboración propia.

\section{Lucha por la estabilidad laboral}

La estabilidad laboral es el anhelo de la gran mayoría de la población trabajadora de la UNA, sin embargo esta condición no es tan simple de alcanzar, en tanto, persisten elementos que tienden a influir en el camino laboral para obtener la propiedad. En el marco de lo anterior, la estabilidad laboral no solo podría asociarse a la denominación dicotómica del ser o no propietario, ya que a su vez se enlaza con las condiciones laborales y las posibilidades de crecimiento laboral y profesional, en tanto es posible denotar diferencias en estos aspectos dentro de la

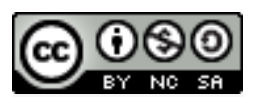

La Revista Estudios es editada por la Universidad de Costa Rica y se distribuye bajo una Licencia Creative Commons Atribución-NoComercial-CompartirIgual 3.0 Costa Rica. Para más información envíe un mensaje a revistaestudios.eeg@ucr.ac.cr. 
Dossier: Mujeres y Educación en la Universidad Nacional

Red de Mujeres Investigadoras de la Universidad Nacional (UNA)

misma población interina, tal es el caso de las mujeres que han sido contratadas de esta forma.

En cuanto a un aspecto tan relevante como la posibilidad de contar con nombramiento anualizado, tal y como lo refleja la figura 2 , un $77,1 \%$ de la población femenina tiene un nombramiento anualizado respecto a un $66,7 \%$ de la población masculina. Con respecto a esto es necesario indicar tal como se señaló anteriormente que en la población de funcionarias, un $40 \%$ ocupa cargos administrativos, a diferencia de la población de funcionarios en la que tan solo un $5,1 \%$ ocupa este tipo de cargos; que usualmente tienen la posibilidad de laborar a tiempo completo y de forma anualizada.

En el caso de las funcionarias un $100.0 \%$ de las administrativas cuenta con un nombramiento anualizado mientras que en el caso de las académicas alcanza un porcentaje menor de $61,9 \%$, lo anterior muestra que las mujeres en puestos administrativos, cuentan con mayor estabilidad en sus ingresos económicos y posibilidad de sufragar sus gastos sin interrupciones en los contratos de trabajo. Esta situación también contribuye a disminuir el sentimiento de zozobra e inseguridad cuando los nombramientos son por ciclos lectivos, condición que enfrenta una población importante de académicas/os.

\section{(c) (i) (9)}

La Revista Estudios es editada por la Universidad de Costa Rica y se distribuye bajo una Licencia Creative Commons Atribución-NoComercial-CompartirIgual 3.0 Costa Rica. Para más información envíe un mensaje a 
Dossier: Mujeres y Educación en la Universidad Nacional

Red de Mujeres Investigadoras de la Universidad Nacional (UNA)

Figura 2. Distribución porcentual de la población censada, según sexo y

disponibilidad de Nombramiento anualizado.

\section{Femenino $77,1 \%$}

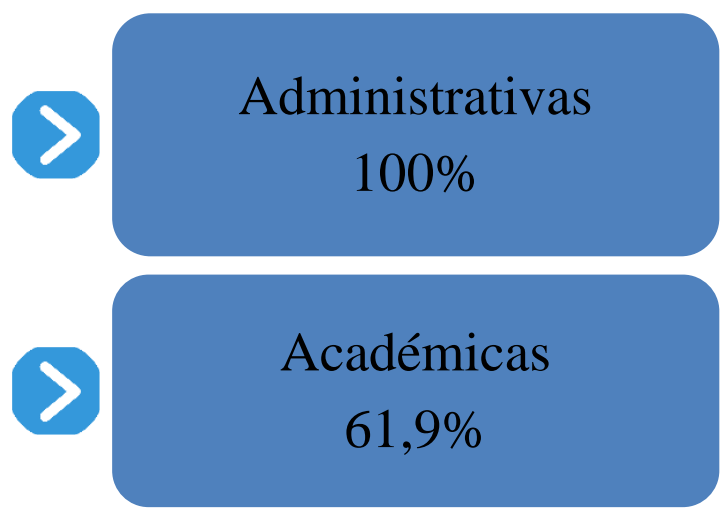

Masculino $66,7 \%$

Fuente: IDESPO-UNA. Censo Percepciones sobre las condiciones de interinazgo en la Universidad Nacional, 2020. Elaboración propia.

Respecto a la jornada laboral actual en la Universidad Nacional, los datos dan a conocer que un $88,6 \%$ de las mujeres censadas se encuentran nombradas tiempo completo, mientras que un $69,2 \%$ de los hombres censados está en la misma condición. En el caso particular de las funcionarias un $100.0 \%$ de las administrativas laboran jornada completa, por otra parte en las académicas se da en un porcentaje menor, correspondiente a un $81,0 \%$, estos datos guardan relación con lo señalado respecto al nombramiento anualizado.

\section{(C) $(\Theta \odot$}

La Revista Estudios es editada por la Universidad de Costa Rica y se distribuye bajo una Licencia Creative Commons Atribución-NoComercial-CompartirIgual 3.0 Costa Rica. Para más información envíe un mensaje a revistaestudios.eeg@ucr.ac.cr. 
Dossier: Mujeres y Educación en la Universidad Nacional

Red de Mujeres Investigadoras de la Universidad Nacional (UNA)

Tabla 4. Distribución porcentual de la jornada laboral actual de la población censada en la Universidad Nacional, 2020.

\begin{tabular}{|r|r|r|}
\hline Jornada & Femenino & Masculino \\
\hline $1 / 4$ tiempo & $2,9 \%$ & $5,1 \%$ \\
\hline $1 / 2$ tiempo & $8,6 \%$ & $25,6 \%$ \\
\hline Tiempo Completo & $88,6 \%$ & $69,2 \%$ \\
\hline Total & $\mathbf{1 0 0 , 0} \%$ & $\mathbf{1 0 0 , 0} \%$ \\
\hline
\end{tabular}

Fuente: IDESPO-UNA. Censo Percepciones sobre las condiciones de interinazgo en la Universidad Nacional, 2020. Elaboración propia.

Tabla 5. Distribución porcentual de la jornada laboral actual de las funcionarias censadas en la Universidad Nacional, según tipo de cargo, 2020.

\begin{tabular}{|l|r|r|}
\hline Jornada & Administrativas & Académicas \\
\hline Tiempo parcial & & $19,0 \%$ \\
\hline Tiempo completo & $100,0 \%$ & $81,0 \%$ \\
\hline Total & $\mathbf{1 0 0 , 0} \%$ & $\mathbf{1 0 0 , 0} \%$ \\
\hline
\end{tabular}

Fuente: IDESPO-UNA. Censo Percepciones sobre las condiciones de interinazgo en la Universidad Nacional, 2020. Elaboración propia.

En referencia a haber concursado por un puesto en propiedad en la UNA la población participante en el censo indica que un $48,7 \%$ de funcionarios han concursado contrario a un $40,0 \%$ de funcionarias, lo que denota que parece existir una mayor propensión por parte de los hombres a participar en este tipo de procesos laborales.

\section{(C) $\odot \Theta($}

La Revista Estudios es editada por la Universidad de Costa Rica y se distribuye bajo una Licencia Creative Commons Atribución-NoComercial-CompartirIgual 3.0 Costa Rica. Para más información envíe un mensaje a revistaestudios.eeg@ucr.ac.cr. 
Dossier: Mujeres y Educación en la Universidad Nacional

Red de Mujeres Investigadoras de la Universidad Nacional (UNA)

Del 40,0\% de funcionarias a nivel general, la distribución porcentual según cargo que ocupan se comporta de la siguiente manera. En el grupo de las administrativas el $42,9 \%$ han concursado por un puesto en propiedad; en el grupo de las académicas el porcentaje es menor, siendo de un 38,1\%, esto refleja una mayor oportunidad en el sector administrativo para participar en concursos dado que tienden a presentarse con más frecuencia que en el sector académico.

Figura 3. Distribución porcentual de la población censada que ha concursado por un puesto en propiedad en la UNA según sexo, 2020.

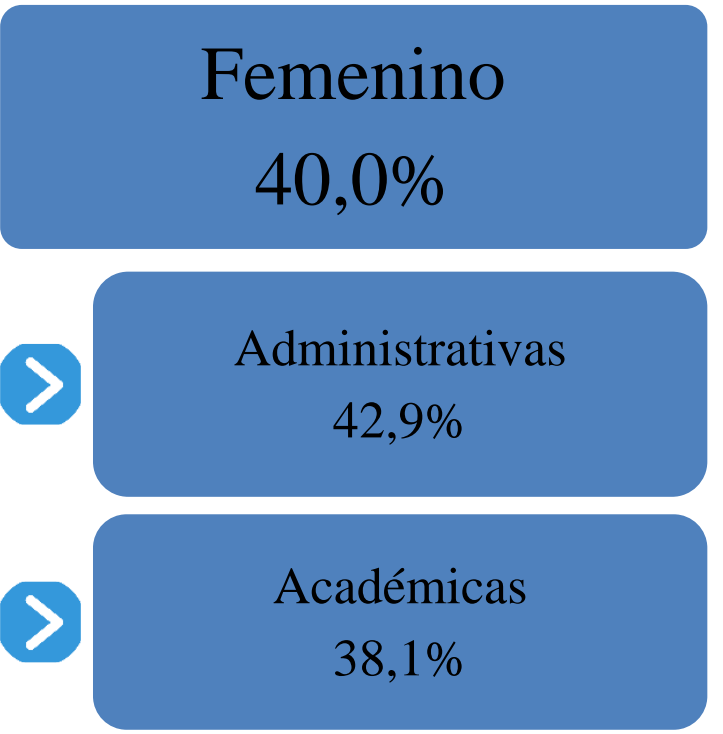

\section{Masculino $48,7 \%$}

Fuente: IDESPO-UNA. Censo Percepciones sobre las condiciones de interinazgo en la Universidad Nacional, 2020. Elaboración propia.

\section{(ㅇ) $\odot \odot$}

La Revista Estudios es editada por la Universidad de Costa Rica y se distribuye bajo una Licencia Creative Commons Atribución-NoComercial-CompartirIgual 3.0 Costa Rica. Para más información envíe un mensaje a revistaestudios.eeg@ucr.ac.cr. 
Dossier: Mujeres y Educación en la Universidad Nacional

Red de Mujeres Investigadoras de la Universidad Nacional (UNA)

\section{Crecimiento profesional de la población femenina interina}

Cuando se considera el crecimiento profesional de las mujeres, es indiscutible la necesidad de abordar el género como categoría analítica; al respecto Osorio (2013), en referencia Amigot (2005); Izquierdo (1985) manifiesta que: "El uso del género como aproximación analítica, nos permite identificar aquellos modelos preestablecidos que limitan nuestras potencialidades e intereses en las prácticas cotidianas" (2013, p. 39). Es decir se asocia a todas las características y normas que se han establecido para las mujeres en el marco de una sociedad patriarcal que castra las posibilidades de desarrollarse fuera del ámbito claramente demarcado del deber ser desde la visión tradicional del género.

Al respecto, la información relativa a la categoría académica actual de la población femenina señala que la mayoría de mujeres se encuentran ubicadas en las categorías de menor rango (Profesor Instructor Licenciado y Profesor 1), y siendo sumamente significativo que ninguna de las censadas ostenta actualmente la categoría de Catedrática; caso contrario a los académicos que sustentan, en mayor grado, los puestos de Profesor 2, Profesor 2 con incentivo y Catedrático, lo que implica un incentivo económico mejor y reconocimiento profesional que el que están percibiendo las mujeres. Esta realidad, podría estar vinculada con las dificultades que tienen muchas funcionarias para producir académicamente debido a las múltiples tareas domésticas y de cuido que demandan las estructuras familiares de parte de ellas, y que por consiguiente deben atender a la luz de lo que el sistema patriarcal les delega.

\section{(C) $(0 \odot$}

La Revista Estudios es editada por la Universidad de Costa Rica y se distribuye bajo una Licencia Creative Commons Atribución-NoComercial-CompartirIgual 3.0 Costa Rica. Para más información envíe un mensaje a 
Dossier: Mujeres y Educación en la Universidad Nacional

Red de Mujeres Investigadoras de la Universidad Nacional (UNA)

Tabla 6. Distribución porcentual de la categoría académica actual de la población censada, según sexo 2020.

\begin{tabular}{|l|r|r|}
\hline \multicolumn{1}{|c|}{ Profesor Instructor Licenciado } & Femenino & Masculino \\
\hline Profesor 1 & $38,1 \%$ & $18,9 \%$ \\
\hline Profesor 2 & $33,3 \%$ & $29,7 \%$ \\
\hline Profesor 2 con incentivo & $9,5 \%$ & $21,6 \%$ \\
\hline Catedrático & $19,0 \%$ & $21,6 \%$ \\
\hline & & $8,1 \%$ \\
\hline
\end{tabular}

Fuente: IDESPO-UNA. Censo Percepciones sobre las condiciones de interinazgo en la Universidad Nacional, 2020. Elaboración propia.

Lo anterior, guarda relación con los datos en cuanto al último grado académico aprobado por las mujeres censadas, tanto administrativas como académicas, esta información indica que un 2,9\% no completaron la secundaria, 11,4\% completaron la licenciatura y $68,6 \%$ cuentan con una maestría y ninguna tiene un doctorado. En el caso de los hombres, 2,6\% completaron la secundaria, $74,4 \%$ tienen un título de maestría y un $15,4 \%$ tienen un doctorado. Estos datos refieren que el grupo de hombres, contrario a las mujeres, han alcanzado mayores niveles académicos.

Es así, como las mujeres al tener un menor reconocimiento académico y profesional, aunado a las condiciones impuestas por el sistema patriarcal como propias de su género, las hace proclives a ocupar espacios que las ubican en condiciones de subordinación, en relación a esto Osorio, señala: "La división sexual del trabajo ha establecido determinados lugares a hombres y mujeres, prescribiendo

\section{(ब) $(\Theta \odot$}

La Revista Estudios es editada por la Universidad de Costa Rica y se distribuye bajo una Licencia Creative Commons Atribución-NoComercial-CompartirIgual 3.0 Costa Rica. Para más información envíe un mensaje a revistaestudios.eeg@ucr.ac.cr. 
Dossier: Mujeres y Educación en la Universidad Nacional

Red de Mujeres Investigadoras de la Universidad Nacional (UNA)

prácticas y naturalizando espacios de dominación. En particular, ello ha implicado colocar a las mujeres en situación de subordinación" (Osorio, 2013, p. 39).

Tabla 7. Distribución porcentual de la población censada según último grado académico aprobado, 2020.

\begin{tabular}{|l|r|r|}
\hline & Femenino & Masculino \\
\hline Secundaria incompleta & $2,9 \%$ & $2,6 \%$ \\
\hline Secundaria completa & & $2,6 \%$ \\
\hline Diplomado/Técnico & $5,7 \%$ & \\
\hline Bachillerato universitario incompleto & $5,7 \%$ & $5,1 \%$ \\
\hline Bachillerato universitario completo & $5,7 \%$ & $74,4 \%$ \\
\hline Licenciatura & $11,4 \%$ & $15,4 \%$ \\
\hline Maestría & $68,6 \%$ & $\mathbf{1 0 0 , 0} \%$ \\
\hline Doctorado & & $\mathbf{1 0 0 , 0} \%$ \\
\hline Total & & \\
\hline
\end{tabular}

Fuente: IDESPO-UNA. Censo Percepciones sobre las condiciones de interinazgo en la Universidad Nacional, 2020. Elaboración propia.

En concordancia con lo anterior, resulta relevante indagar acerca de los estudios académicos que realizan actualmente, los datos obtenidos indican que $74,3 \%$ de funcionarias no realizan estudios, $2.9 \%$ están cursando una maestría, 5,7\% se encuentran en un doctorado y algunas mujeres, en menor porcentaje, realizan estudios de diplomado, licenciatura, entre otros. En el caso de los hombres, 61,5\% no están estudiando, 15,4\% realizan estudios de maestría y $20,5 \%$ están en un doctorado. Estos datos evidencian que los funcionarios se encuentran en mayor porcentaje efectuando estudios de posgrado.

\section{(ब) $\odot \otimes(\odot$}

La Revista Estudios es editada por la Universidad de Costa Rica y se distribuye bajo una Licencia Creative Commons Atribución-NoComercial-CompartirIgual 3.0 Costa Rica. Para más información envíe un mensaje a revistaestudios.eeg@ucr.ac.cr. 
Dossier: Mujeres y Educación en la Universidad Nacional Red de Mujeres Investigadoras de la Universidad Nacional (UNA)

Tabla 8. Distribución porcentual de la población censada según nivel de Estudios cursados actualmente, 2020.

\begin{tabular}{|l|r|r|}
\hline & Femenino & Masculino \\
\hline No estudia & $74,3 \%$ & $61,5 \%$ \\
\hline Secundaria completa & $2,9 \%$ & $2,6 \%$ \\
\hline Diplomado/ Técnico & $2,9 \%$ & \\
\hline Bachillerato universitario & $5,7 \%$ & $15,4 \%$ \\
\hline Licenciatura & $5,7 \%$ & $20,0 \%$ \\
\hline Maestría & $2,9 \%$ & $100,0 \%$ \\
\hline Doctorado & $5,7 \%$ & $100,0 \%$ \\
\hline Total & $10 \%$ & \\
\hline
\end{tabular}

Fuente: IDESPO-UNA. Censo Percepciones sobre las condiciones de interinazgo en la Universidad Nacional, 2020. Elaboración propia

En lo que respecta a las funciones que se ejercen en la Universidad Nacional, resalta el hecho de que el $100,0 \%$ de académicas son docentes, $23,8 \%$ realizan tareas de investigación, un $23,8 \%$ son extensionistas, 2,9\% participan en la edición de una revista y sólo un 5,7\% son coordinadoras de carrera. En el caso de los académicos $94,6 \%$ son docentes, $35,1 \%$ realizan investigación, $16,2 \%$ son extensionistas y también participan en comisiones de trabajo, puestos de subdirección, Consejo Académico y coordinación de carrera, espacios donde se toman decisiones respecto al quehacer académico y administrativo de las unidades académicas.

\section{(ब) $\odot \otimes(\odot$}

La Revista Estudios es editada por la Universidad de Costa Rica y se distribuye bajo una Licencia Creative Commons Atribución-NoComercial-CompartirIgual 3.0 Costa Rica. Para más información envíe un mensaje a revistaestudios.eeg@ucr.ac.cr. 
Dossier: Mujeres y Educación en la Universidad Nacional

Red de Mujeres Investigadoras de la Universidad Nacional (UNA)

Resulta interesante que las mujeres del estudio tienden a ejercer funciones más en cargos/roles de enseñanza, tradicionalmente delegados en el género femenino, y los hombres participan con mayor frecuencia en puestos de dirección y conducción académica. En este sentido Gaete-Quezada (2015) indica que para Sanders et al. (2009), el techo de cristal que enfrentan las mujeres para alcanzar altos cargos en las organizaciones, puede ser causado por:

"La tradicional división de tareas domésticas y las responsabilidades del cuidado de los hijos; la incompatibilidad de los roles familiares y profesionales; baja autoestima de las mujeres; el sexismo y la discriminación de género manifiesta; el sesgo en los procedimientos de reclutamiento y selección; entre otras" (p. 10).

Tabla 9. Distribución porcentual de las Funciones que se ejercen en la Universidad Nacional la población censada, 2020.

\begin{tabular}{|l|r|r|}
\hline Funciones & Femenino & Masculino \\
\hline Docencia & $100,0 \%$ & $94,6 \%$ \\
\hline Investigación & $23,8 \%$ & $35,1 \%$ \\
\hline Extensión & $23,8 \%$ & $16,2 \%$ \\
\hline Comisiones & & $5,1 \%$ \\
\hline Subdirección & & $2,6 \%$ \\
\hline Consejo Académico & & $2,6 \%$ \\
\hline Coordinación de carrera & $5,7 \%$ & $7,7 \%$ \\
\hline Revista & $2,9 \%$ & \\
\hline
\end{tabular}

Fuente: IDESPO-UNA. Censo Percepciones sobre las condiciones de interinazgo en la Universidad Nacional, 2020. Elaboración propia

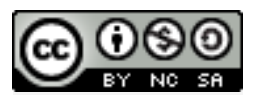

La Revista Estudios es editada por la Universidad de Costa Rica y se distribuye bajo una Licencia Creative Commons Atribución-NoComercial-CompartirIgual 3.0 Costa Rica. Para más información envíe un mensaje a revistaestudios.eeg@ucr.ac.cr. 
Dossier: Mujeres y Educación en la Universidad Nacional Red de Mujeres Investigadoras de la Universidad Nacional (UNA)

\section{Consideraciones finales}

Los resultados del censo muestran, a nivel general, la dificultad que enfrentan las funcionarias académicas para desarrollar tareas en el área de la investigación, lo que se observa como una limitación para ascender en carrera académica. En el caso de las funcionarias administrativas se encuentra que la mayoría ocupa cargos no profesionales, lo que repercute en sus salarios y crecimiento académico. Los datos reflejan que en el caso de las administrativas, se presenta con mayor regularidad la posibilidad de concursar por un puesto en propiedad contrario a lo que viven las académicas.

El estudio evidencia que de la población participante ningún de las funcionarias cuenta con la categoría académica de catedrática ni con un doctorado, contrario a los funcionarios académicos, situación que podría estar determinada por las múltiples tareas que deben atender las mujeres producto de los roles asignados por el sistema patriarcal, que dificultan, para algunas de ellas, seguir estudiando y producir académicamente.

En cuanto a los estudios que cursan actualmente existe un mayor porcentaje de funcionarios realizando estudios de maestría y doctorado, contrario a la situación de las mujeres funcionarias donde un $74,3 \%$ no están cursando estudios en la actualidad. En lo referente a la jornada laboral la totalidad de funcionarias administrativas cuenta con un nombramiento de tiempo completo, situación que no se presenta en el caso de las académicas lo que las hace vivir con mayor frecuencia la inestabilidad y zozobra que el ser "no propietario" implica.

La condición de interinazgo, como contratación laboral, conlleva inestabilidad, incertidumbre y vulnerabilidad en lo personal y en el crecimiento profesional, a esta realidad se adiciona, en el caso de las funcionarias, las limitaciones estructurales

\section{(C) $(0 \bigcirc$}

La Revista Estudios es editada por la Universidad de Costa Rica y se distribuye bajo una Licencia Creative Commons Atribución-NoComercial-CompartirIgual 3.0 Costa Rica. Para más información envíe un mensaje a 
Dossier: Mujeres y Educación en la Universidad Nacional

Red de Mujeres Investigadoras de la Universidad Nacional (UNA)

que el sistema patriarcal impone en el género femenino, lo que expone a estas mujeres a una doble vulnerabilidad. A partir de los resultados de este censo resulta de fundamental importancia que en la Universidad Nacional se abran espacio de discusión respecto a la realidad que vive la comunidad interina para establecer disposiciones que les permita contar con estabilidad laboral que, a su vez, promueva un mayor crecimiento profesional y personal de la población trabajadora universitaria.

\section{Referencias}

Alfaro Esquivel, I., Alvarado Arias, A. (2012). Análisis de los procesos de atracción, selección, y vinculación del personal académico interino regular de la Escuela de Administración de la Universidad Nacional durante los años 2009, 2010 y 2011.(Tesis de Licenciatura). Universidad Nacional, Campus Omar Dengo, Costa Rica

Fernández Carvajal, D., Jiménez Mata, S., Marenco Marrocchi, L., Sequeira Rovira., P. (2014). Diagnóstico Institucional: Relaciones de equidad entre mujeres y hombres, en la Universidad Nacional. Universidad Nacional. Recuperado de www.iem.una.ac.cr/.../diagnostico/.../1-serie-investigaciones-iem

Gaete Quezada, Ricardo. (2015). El techo de cristal en las universidades estatales chilenas. Un análisis exploratorio. Revista iberoamericana de educación superior, 6(17), 3-20. https://dx.doi.org/10.1016/j.rides.2015.06.001

\section{(c) (i) (2)}

La Revista Estudios es editada por la Universidad de Costa Rica y se distribuye bajo una Licencia Creative Commons Atribución-NoComercial-CompartirIgual 3.0 Costa Rica. Para más información envíe un mensaje a revistaestudios.eeg@ucr.ac.cr. 
Dossier: Mujeres y Educación en la Universidad Nacional

Red de Mujeres Investigadoras de la Universidad Nacional (UNA)

Gamboa Jiménez, A. (2015). Entre el desgaste y la precarización laboral: un estudio de la academia interina de la Universidad Nacional. (Tesis de Doctorado). Universidad Nacional, Campus Omar Dengo, Costa Rica.

IDESPO-UNA. Base de datos Censo Percepciones sobre las condiciones de interinazgo en la Universidad Nacional, 2020

Osorio Cabrera, Daniela. (2013). De la división sexual del trabajo hacia la redefinición de las prácticas de cuidado: una experiencia de Economía Solidaria en Cataluña. Summa psicológica UST, 10(1), 37-47. Recuperado el 20 de febrero de 2020, de http://pepsic.bvsalud.org/scielo.php?script=sci_arttext\&pid=S0719$448 \times 2013000100004 \& \operatorname{lng}=p t \& t \operatorname{lng}=e s$.

Segura Espinoza, G. (2014). Casos denunciados en la Universidad Nacional de acoso laboral y psicológico durante 2008 y 2009: Un análisis de sus manifestaciones y consecuencias desde una perspectiva de género. (Tesis de Maestría). Universidad Estatal a Distancia, Costa Rica.

\section{(C) $(0 \odot$}

La Revista Estudios es editada por la Universidad de Costa Rica y se distribuye bajo una Licencia Creative Commons Atribución-NoComercial-CompartirIgual 3.0 Costa Rica. Para más información envíe un mensaje a revistaestudios.eeg@ucr.ac.cr. 